\title{
Application of artificial neural network technology to predicting small faults and folds in coal seams, China
}

\author{
Yifan Zeng $^{1} \cdot$ Shouqiang Liu ${ }^{1} \cdot$ Wei Zhang ${ }^{1} \cdot$ Yanliang Zhai $^{1}$
}

Received: 1 November 2015/Accepted: 15 March 2016/Published online: 30 March 2016

(C) Springer International Publishing Switzerland 2016

\begin{abstract}
Small geologic structures pose a great threat to production safety of the coal mines in China. Many water hazards and rock collapses are related to these small geologic structures. Accurate prediction of these structures relies on multiple lines of evidence including coal seam dip, thickness change, amount of gas accumulated, water flow changes, temperature, fracture type and degree of fragmentation of coal seams. Through the use of artificial neural network technology, this article presents a working method for forecasting small geologic structures in coal mines. The methods are applied to Zhangcun Coal Mine, China. A nonlinear model consisting of coal seam dip and thickness is constructed to predict the small structures in the front of working faces. The predictions are verified by field data. The distribution characteristics of the small structures can be accurately predicted in the coal seam extraction process as long as data of the controlling factors are accurately collected.
\end{abstract}

Keywords Size-limited geologic structure $\cdot$ Artificial neural network $\cdot$ Water hazards $\cdot$ Coal mines, China

\section{Introduction}

Small geologic structures, also referred to as sized-limited structures, are faults and folds of less than $3 \mathrm{~m}$ and are not readily recognizable in individual roadways or working faces (Wang et al. 1993). High frequency of their

Yifan Zeng

zengyifan_1011@sina.com

1 China University of Mining and Technology, Beijing 100083, China occurrence leads to compromised stability of roof and floor in coal mines, resulting in difficulties in roof and floor management. In coal mines that are susceptible to gas outbursts, the outbursts often occur in places where the small geologic structures are intensely developed. These small structures often form pathways for groundwater, inducing significant increase of inflow in the mines, even causing water inrushes and flooding mines or working stopes. With the continuous increase in mechanic mining and hydraulic support joint unit use, the production safety issues within the stope caused by the small structures become more serious. However, predicting the small structures is extremely difficult because their hidden nature and limited scale challenge this effort (Li and Ma 1993).

The geologic structures with displacements similar to the thickness of coal seams are not readily identifiable during the phase of coal exploration. Previous studies on the prediction of the small geologic structures mainly focused on two methods-one is geophysical prospecting and the other is application of regional tectonic system analysis. Geophysical methods have often been used for exploring and predicting the structures, such as electrical and seismic wave methods and ground penetrating radar. The radio wave method is currently the most popular and widely used in situ geophysical method. The rock layers are fractured/faulted during the tectonic deformation. Adjacent to the geologic structure influence area, many different fractured/faulted faces exist. These faces deflect, reflect or scatter the radio waves; thus, the absorbed energy is larger than the normal area. If the fracture/fault zones are full of water, the electrical resistivity becomes lower and the absorption coefficient becomes larger. Therefore, these fracture/fault zones result in the abnormal values on the curves of geophysical prospecting. Another method for small geologic structure prediction is the analysis of the 
regional tectonic system. The distribution of small geologic structures is under control of the regional tectonic system, with which most of the structures are associated. Some of the geologic structures are small faults along coal seam planes under the area stress, and some of them in the coal seam are formed under the local stress field. To predict these structures, the distribution of regional large/median tectonic system must be determined first. Attention must be paid for mining approaches of these tectonic areas (Yang 1997). Although the methods aforementioned have exerted huge effects in the prediction of the small geologic structures, they have their individual deficiencies. They are costly and often misjudge the position of these structures by application of the analysis of the regional large/median tectonic system (Wu et al. 2007, 2008).

The challenges in predicting the small geologic structures include their limited sizes and lack of obvious expression on the subsurface. However, knowledge about these structures has been accumulated during the long-term mining practices. The following factors are typically indicative of the existence of small structures (Wu et al. 1999):

- Thickness of coal seam: tectonic movement is a major cause of change in coal seam thickness. Based on statistics, there may exist small structures in the forward direction when the change in coal seam thickness is greater than $20 \%$. Some faults bifurcate and causes pinch-out in the coal seams, whereas folds often accompany irregular seams with mutated thickness.

- Dip of coal seam: small folds and faults tend to pull the coal beds, even crumple and deform them. Practical measurements indicate that small structures may exist in the forward direction where the coal seam dip angles more than $0.6^{\circ}$ within less than $10 \mathrm{~m}$.

- Degree of fragmentation in the coal seam: rigid deformation results in fractures in coal seams that are fragmented, although the original layering structures may remain.

- Fracture type: fractures in coal seams can be divided into endogenous fractures and exogenous fractures. Exogenous fractures are produced by tectonic activities after the coal seams are formed. Endogenous fractures are characterized by perpendicular to bedding orientation and smooth surfaces. Exogenous fractures may intersect with coal layers in any angle and have wavy surfaces with feathery scratches. When the seam fracture has the above changes, there may exist small structures in the front of the mining direction $(\mathrm{Wu}$ et al. 2011).

- Gas content in coal seam: originally, gas content in coal seams is typically big, but the amount of gas emission is usually small and slow. When the geologic structure is developed, the gas content of coal seams is significantly reduced (Wu et al. 2015).

- Temperature of coal seam: small geologic structures may become water-bearing media or pathways for groundwater flow, which may lead to a change in the temperature of the coal seam.

- Water discharge from the coal seam: the geologic structures are the most important channels for water inrush. When mining areas approach fault zones, water inflow may increase and water inrushes may occur. Abnormal increase of inflow at the forward direction of digging may be indicative of the presence of small geologic structures (Zeng and $\mathrm{Wu} 2014$ ).

The fact that there are multiple factors relevant to the small geologic structures and the weighting coefficient for each factor is not clear makes artificial neural network (ANN) an appropriate approach to predicting these structures. The ANNs have the abilities of the massively distributed parallel processing function, which has a natural propensity for storing experiential knowledge, especially when it is necessary to deal with several factors whose relation to the prediction object is not clear.

\section{ANN-based small structure prediction model}

ANN has been extensively used in earth sciences including geophysics exploration, iron cap recognition, and oil and gas discrimination (McCorak 1991; Cai et al. 1994; Lv and Zhao 1998). ANN brings a new approach to predicting the geologic structures without the necessity of prior knowledge of the relationships between the various affecting factors. Model training is carried out automatically using known small structures and their affecting factors, which can avoid difficulty in data analysis and data processing. With the lines of evidence for small geologic structures as the inputs and known small structures as the output, the focus is on the factors in favor of the existence of small structures (Wang 1995; Chen and Wu 2001). The basic principle of ANN is well documented (Wu et al. 1999; Cai et al. 1994) and application of such models often consists of the following three steps:

1. Provide the necessary input and training condition for ANNs with a number sets of data relevant to the structures. The data collections should be carried out in the failure zone, affected zone and normal zone. These zones have been observed from the numerous coal laneways.

2. Apply the ANN analysis model to deal with the data and determine the weighting coefficient for the individual factor by adopting the non-linear mathematics method; 
3. Establish the overlap model and mathematic formula for the prediction of the small geologic structures.

ANN can automatically simulate the natural relationship between the various factors, get a global optimization calculation, reduce man-made interference and improve the effectiveness of the small structure prediction. Once the ANN-based prediction model of small structures is established, it often has a universal use and can be used in mines with various geologic structures (Wu and Ye 2008; Cao and Jiang 2002). In this paper, prediction of the small geologic structures is performed in Zhangcun Coal Mine to show the training and verification processes of the ANNbased model.

\section{Background setting of the Zhangcun Coal Mine}

Zhangcun Coal Mine lies in Xiandewang and Baita Town of Hebei Province, China (Fig. 1). It is on a platform terrain, which is bounded by the hilly country east Taihang Mountains. There is no perennial stream, and only two seasonal creeks flow through this area. Geologically, the study area is characterized by a set of Carboniferous-Permian coal strata, which is typical of North China. The ninth coal seam is a stable layer that can be commercially exploited, while the second, eighth and tenth coal seams are partially exploitable.

Geomorphologically, the Zhangcun Coal Mine is in the central Xingtai Wuan depression, located on the northern side of Ming River. The area is characterized by folds, faults and magmatic rocks. According to the faulted structure framework, Zhangcun Coal Mine is in the western block segment of a horst, which results from Xiandewang normal faults and Zhaodian normal faults. According to the folded structure framework, the coal mine is part of a regional syncline and anticline system. The Zhangcun Coal Mine occupies most of the Zhongguan anticline and Xiaguan-Zhujinzi syncline. In addition, there are many secondary minor folds. Because of the relatively strong Yanshanian magmatic activity, magma intrusions occurred in the mine field, which has a great impact on the coal seams and coal and geological structures (Fig. 2). There are 205 faults reported in the course of exploration and production in the mine. Of these reported faults, 11 are large- and medium-sized faults with their displacements being more than $20 \mathrm{~m}$, accounting for $5.4 \%$ of the revealed faults. Sixty-two are medium- and small-sized faults with displacements between 3 and $20 \mathrm{~m}$, accounting for $30.2 \%$ of the revealed faults. The remaining 132 are small faults with displacements less than $3 \mathrm{~m}$, accounting for $64.4 \%$ of the revealed faults.

The high density of small geologic structures seriously affects roadway tunneling and mining operation in the coal mine. Furthermore, because the Zhangcun Coal Mine is located on the Zhongguan anticline, the shallow aquifer and the fractured karst aquifer co-exist. Mining of the lowest coal seam is threatened by the fractured karst water that is confined and pressurized. As shown in Fig. 3, the ninth coal seam is underlain by the Benxi formation and Ordovician limestone aquifer. The Ordovician limestone is

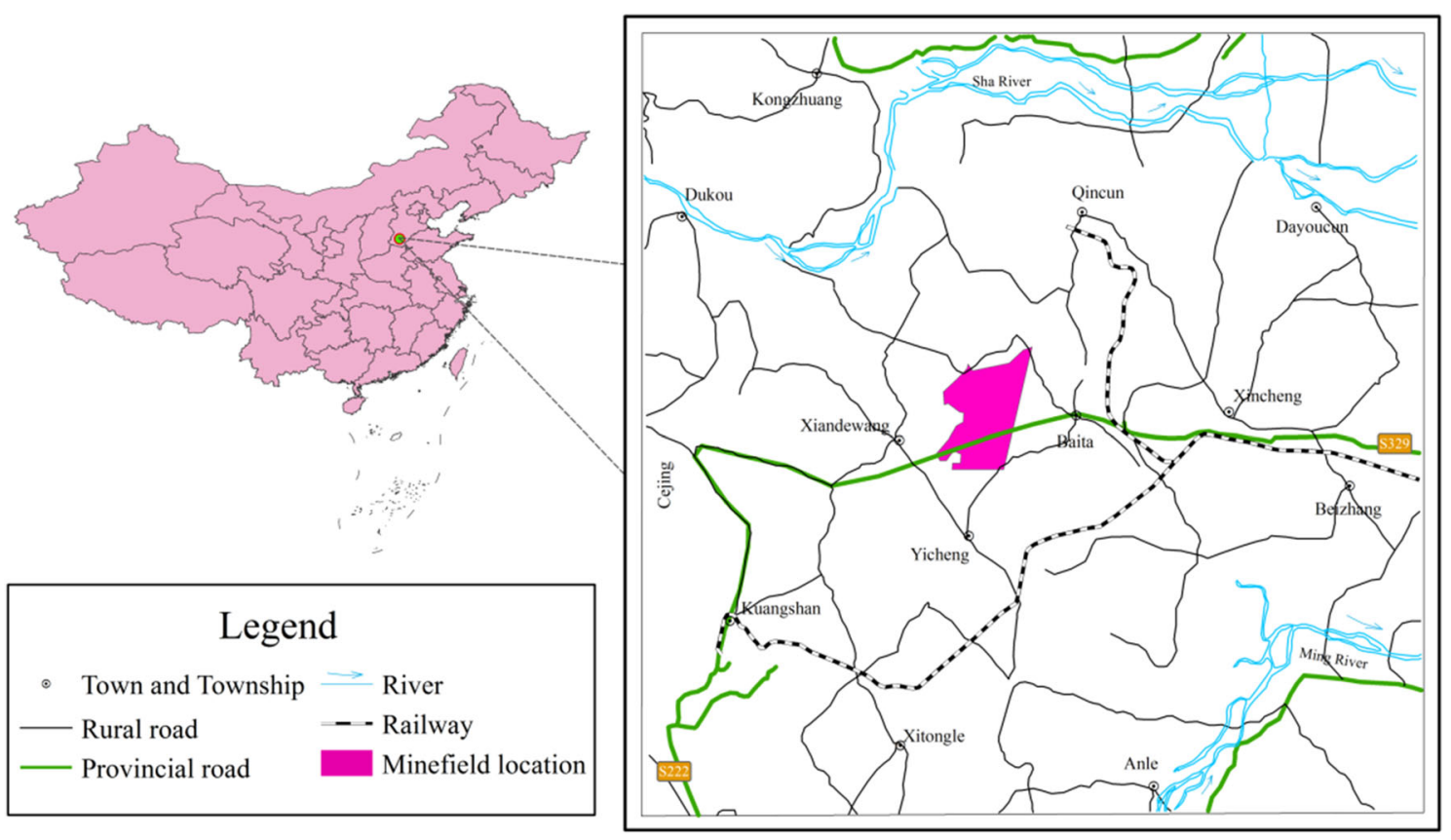

Fig. 1 Location of the Zhangcun Coal Mine 


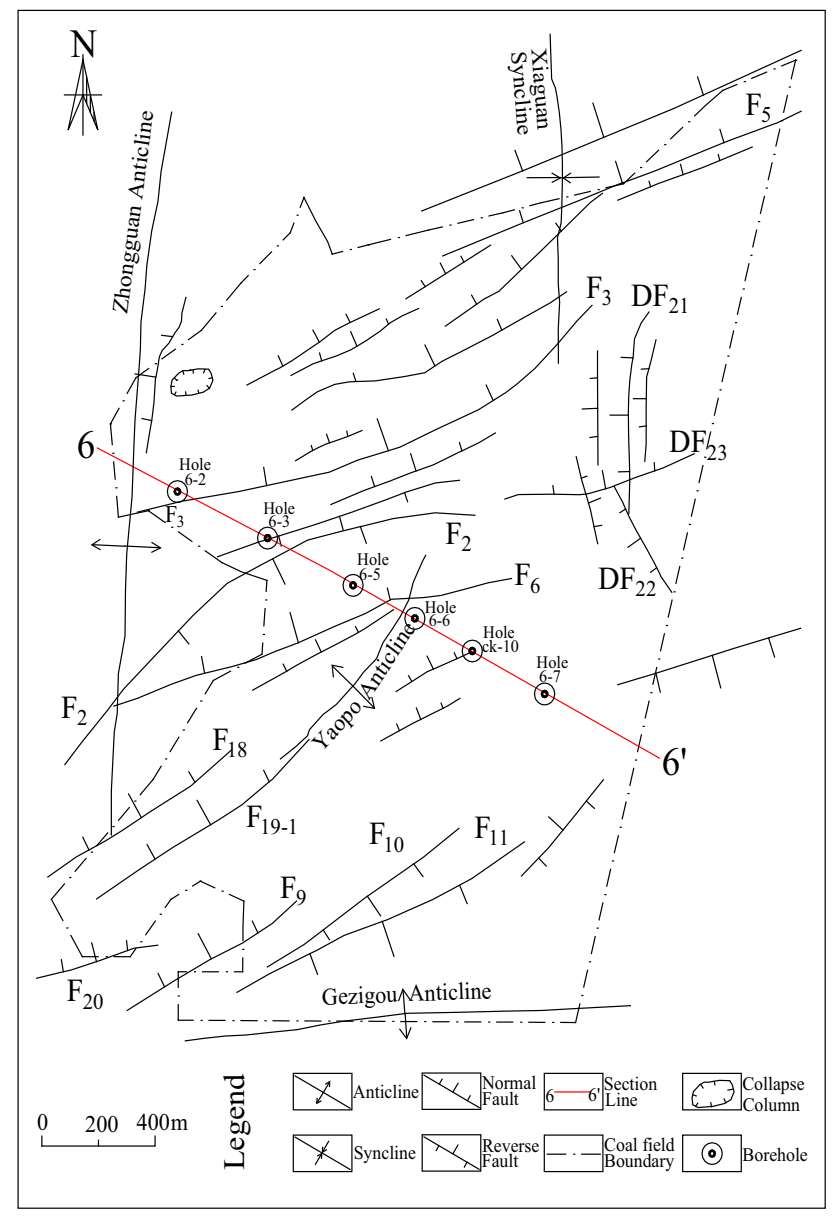

Fig. 2 Geologic structures of the Zhangcun Coal Mine

the main aquifer that poses threat to the safe operations of the mine. The average thickness of the strata between the top of the Ordovician limestone and the bottom of the ninth coal seam is $32 \mathrm{~m}$. Dense distribution of small geologic structures undermines the stability of the coal seam floor and tends to form subtle water channels for water flow. The additional water flow not only increases cost of the mine drainage, but also causes water inrush accidents.

\section{Establishment of the forecasting model and its application}

\section{Date collection and quantification of controlling factors}

As shown in Fig. 4, two factors are selected for the input factors to construct the size-limited structure backpropagation neural network model. The selected factors are the coal seam dip angle (CSDA) (Information source set A) and coal seam thickness change (CSTK) (Information source set B). Simulation training is based on Matlab that establishes the nonlinear relationships of the input and output layers. CSDA and CSTK are training and test data of the ANN model in each area. Twenty-six sets of test data are used as the training and test samples. The first 23 samples are the samples to train the network, while the remaining three examples are test samples for checking the performance of the network.

Because of the wide variation of CSDA and CSTK in different samples, the numerical change rate that is relative to the normal zone is used to be treated in a unified manner. The values from the normal zone act as the baseline to 0 , whereas the values of the affected and failure zones are given, respectively, based on the normalized values. The similarity thresholds for the three zones are presented in Tables 1 and 2. The mathematical expressions are as follows:

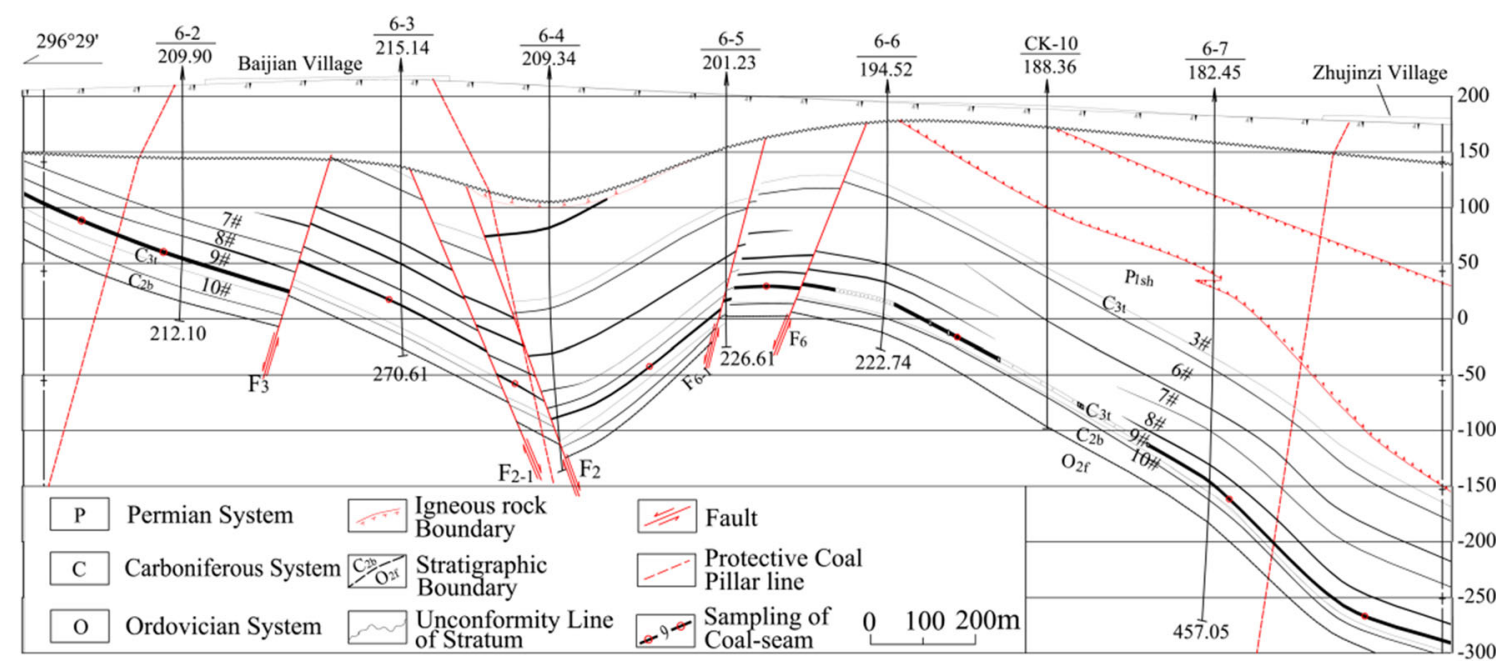

Fig. 3 Geologic cross section of the Zhangcun Coal Mine along the 6-6' (location of 6-6' is shown in Fig. 2) 

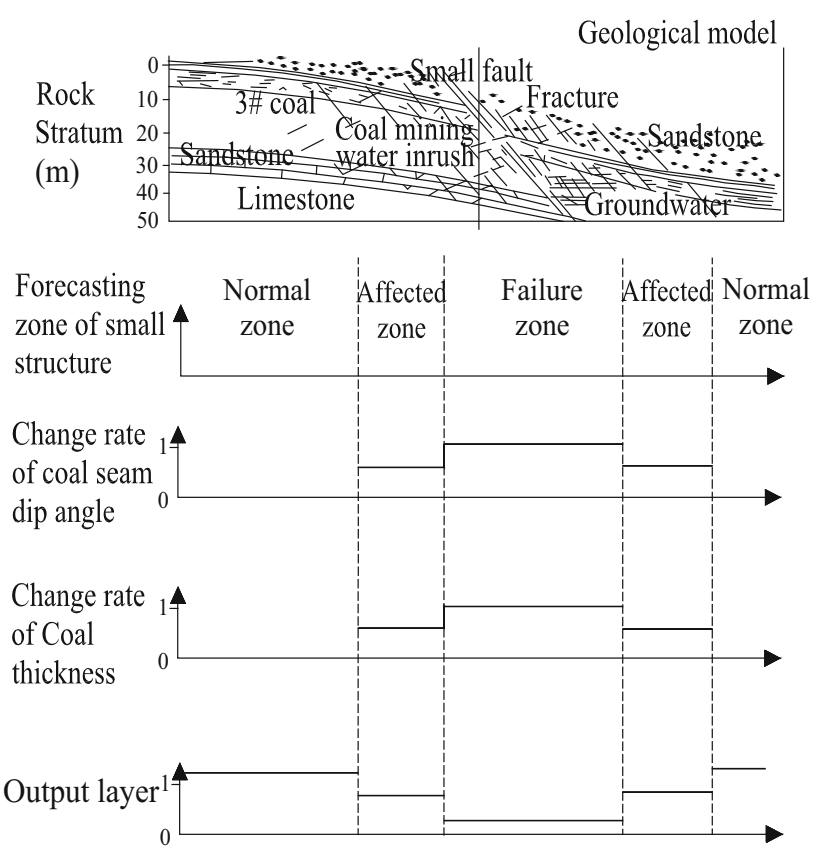

Fig. 4 Schematic relationship between controlling factors and sizelimited structures in the Zhangcun Coal Mine

Table 1 Training data in the normal zone

\begin{tabular}{llll}
\hline Sample & $\begin{array}{l}\text { Change rate of } \\
\text { coal thickness }\end{array}$ & $\begin{array}{l}\text { Change rate of coal } \\
\text { seam dip angle }\end{array}$ & $\begin{array}{l}\text { Expected } \\
\text { output }\end{array}$ \\
\hline $1-23$ & 0.0000 & 0.0000 & $1,0,0$ \\
\hline
\end{tabular}

$y_{1}=|(b-a) / a|, y_{2}=|(c-a) / a|$,

where $y_{1}$ and $y_{2}$ are the normalized data for the affected zone and the failure zone, respectively. Parameters $a, b$ and $c$ are the sample data from the normal zone, affected zone and failure zone. The collected data are shown in Tables 1 through 3 .

\section{Network design}

This research adopts the backpropagation ANN, which is particularly effective in the analysis of complex relationships. Because two main factors are used for prediction of the size-limited structures in the Zhangcun Coal Mine, the small structure prediction evaluation model has two input nodes. The risk levels of small structure in front of working faces are the output of the model. The mine area is divided into three zones: the normal zone, the affected zone and the failure zone, as shown in Fig. 5. Correspondingly, the model has three output nodes in the form of matrix $\{1,0$, $0\},\{0,1,0\}$ and $\{0,0,1\}$ for the normal zone, the affected zone and the failure zone. A single hidden layer is selected in this prediction model because its feedforward neural network can map all continuous functions.

Adding nodes of hidden layers can improve the matching accuracy of the network and training data sets. According to the neuron number of input and output layer, preliminary calculations of neurons in the intermediate layer should be between 9 and 15. Verifying the network performance by graded calculation results in a selection of 15 hidden-layer neurons. The transfer function of sigmoid is adopted from the input layer to the hidden layer, while Purlin transfer function is used from the hidden layer to the output layer and Traimlm function is used as a training function.

\section{Network training}

Matlab is used to perform the simulation training to build the backpropagation ANN. Before the train function is

Table 2 Training data in the affected zone

\begin{tabular}{|c|c|c|c|c|c|c|c|}
\hline Sample & $\begin{array}{l}\text { Change rate of } \\
\text { coal thickness }\end{array}$ & $\begin{array}{l}\text { Change rate of } \\
\text { coal seam dip angle }\end{array}$ & $\begin{array}{l}\text { Expected } \\
\text { output }\end{array}$ & Sample & $\begin{array}{l}\text { Change rate of } \\
\text { coal thickness }\end{array}$ & $\begin{array}{l}\text { Change rate of coal } \\
\text { seam dip angle }\end{array}$ & $\begin{array}{l}\text { Expected } \\
\text { output }\end{array}$ \\
\hline 1 & 0.2381 & 0.7778 & $0,1,0$ & 13 & 0.3308 & 0.8333 & $0,1,0$ \\
\hline 2 & 0.3705 & 0.3333 & $0,1,0$ & 14 & 0.3687 & 0.4359 & $0,1,0$ \\
\hline 3 & 0.2967 & 0.6364 & $0,1,0$ & 15 & 0.8439 & 1.0000 & $0,1,0$ \\
\hline 4 & 0.3705 & 0.5556 & $0,1,0$ & 16 & 0.2976 & 0.2381 & $0,1,0$ \\
\hline 5 & 0.3532 & 0.3333 & $0,1,0$ & 17 & 0.1494 & 0.7037 & $0,1,0$ \\
\hline 6 & 0.3345 & 0.0909 & $0,1,0$ & 18 & 0.6389 & 0.0556 & $0,1,0$ \\
\hline 7 & 0.1072 & 0.0370 & $0,1,0$ & 19 & 0.1346 & 0.5556 & $0,1,0$ \\
\hline 8 & 0.3494 & 0.1579 & $0,1,0$ & 20 & 0.0000 & 0.3333 & $0,1,0$ \\
\hline 9 & 0.3403 & 0.0435 & $0,1,0$ & 21 & 0.3308 & 1.5000 & $0,1,0$ \\
\hline 10 & 0.475 & 0.3623 & $0,1,0$ & 22 & 0.5986 & 0.0000 & $0,1,0$ \\
\hline 11 & 0.4768 & 0.3333 & $0,1,0$ & 23 & 0.5042 & 1.6667 & $0,1,0$ \\
\hline 12 & 0.3345 & 0.4222 & $0,1,0$ & & & & \\
\hline
\end{tabular}


Table 3 Training data in the failure zone

\begin{tabular}{|c|c|c|c|c|c|c|c|}
\hline Sample & $\begin{array}{l}\text { Change rate of } \\
\text { coal thickness }\end{array}$ & $\begin{array}{l}\text { Change rate of coal } \\
\text { seam dip angle }\end{array}$ & $\begin{array}{l}\text { Expected } \\
\text { output }\end{array}$ & Sample & $\begin{array}{l}\text { Change rate of } \\
\text { coal thickness }\end{array}$ & $\begin{array}{l}\text { Change rate of coal } \\
\text { seam dip angle }\end{array}$ & $\begin{array}{l}\text { Expected } \\
\text { output }\end{array}$ \\
\hline 1 & 0.3542 & 0.0000 & $0,0,1$ & 13 & 0.2939 & 0.5000 & $0,0,1$ \\
\hline 2 & 0.1692 & 0.0667 & $0,0,1$ & 14 & 0.3111 & 0.8462 & $0,0,1$ \\
\hline 3 & 0.2292 & 0.3818 & $0,0,1$ & 15 & 0.4537 & 0.0545 & $0,0,1$ \\
\hline 4 & 0.1692 & 0.4667 & $0,0,1$ & 16 & 0.1063 & 0.6000 & $0,0,1$ \\
\hline 5 & 0.2123 & 0.2000 & $0,0,1$ & 17 & 0.4527 & 0.4667 & $0,0,1$ \\
\hline 6 & 0.2906 & 0.5636 & $0,0,1$ & 18 & 0.1472 & 0.3333 & $0,0,1$ \\
\hline 7 & 0.1928 & 0.3333 & $0,0,1$ & 19 & 0.4183 & 1.0000 & $0,0,1$ \\
\hline 8 & 0.3805 & 0.0316 & $0,0,1$ & 20 & 0.0000 & 0.5500 & $0,0,1$ \\
\hline 9 & 0.2856 & 0.513 & $0,0,1$ & 21 & 0.2939 & 0.0000 & $0,0,1$ \\
\hline 10 & 0.4135 & 0.3391 & $0,0,1$ & 22 & 1.0000 & 0.1200 & $0,0,1$ \\
\hline 11 & 0.3615 & 0.0400 & $0,0,1$ & 23 & 0.7353 & 0.2800 & $0,0,1$ \\
\hline 12 & 0.2906 & 0.2267 & $0,0,1$ & & & & \\
\hline
\end{tabular}

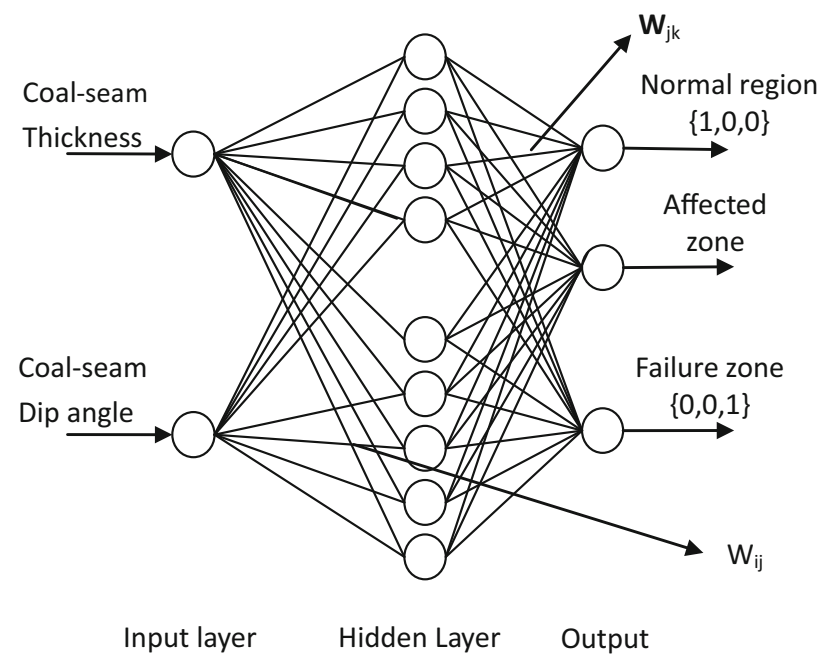

Fig. 5 ANN model for small structure prediction in the Zhangcun Coal Mine

applied onto the network training, set the maximum training period to 1500 , learning rate of the hidden layer and output layer to 0.25 and the training time to 50 . The training accuracy is quantified by $E_{\mathrm{k}}$, the mean square value of global error. The training has met the requirements when the global error variance $E_{\mathrm{k}}$ of network is less than 0.001. Otherwise, we modify the network weights and thresholds between matrix and make iterative approximations until the ideal output conforms to the actual output of the network. The ANN achieves the accuracy requirement with the error mean square $E_{\mathrm{k}}=3.58 \times 10^{-4}$ after 57 steps of training. The convergence curve of the BP network training is shown in Fig. 6.

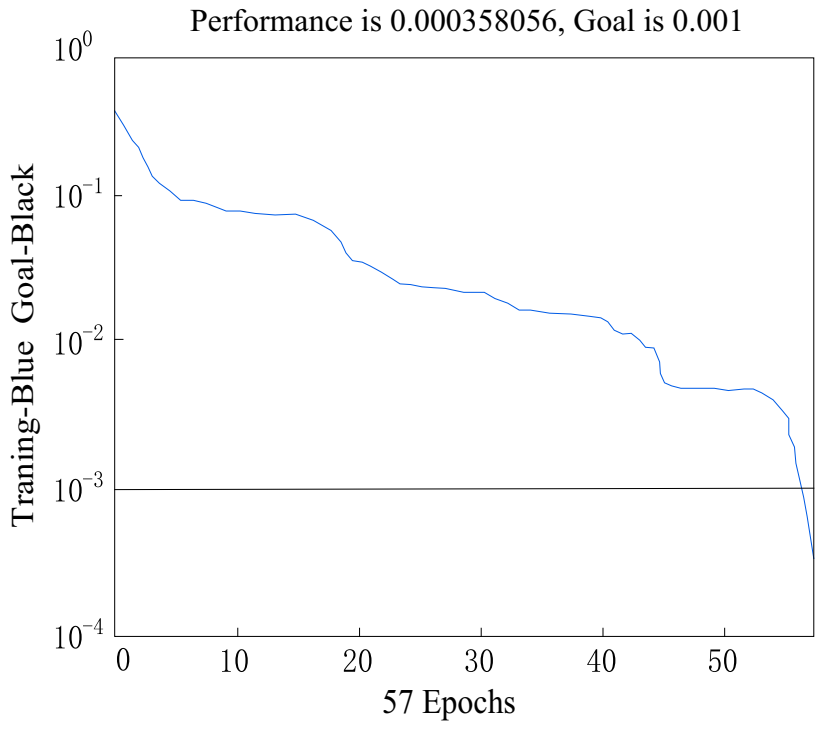

Fig. 6 Convergence process during training

\section{Model validation}

In addition to selecting the 23 sets of samples for the network training, the other three sets of sample are used to test the model's predictive power and verify the network model in predicting small structures. The three sets of sample data are input into the model and comparison is made between the predicted results and field test results. As presented in Table 4, the three sets of actual outputs are close to the expected outputs. The general consistency between the actual output and the expected output verifies the accuracy of the predictive model. The training of the model is considered to be successful. 
Table 4 Comparison of the predicted results and actual measurements

\begin{tabular}{|c|c|c|c|c|c|c|}
\hline \multirow{2}{*}{$\begin{array}{l}\text { Sample } \\
\text { number }\end{array}$} & \multicolumn{2}{|l|}{ Test sample } & \multirow{2}{*}{\multicolumn{2}{|c|}{ Actual output }} & & \multirow[t]{2}{*}{ Expected output } \\
\hline & $\begin{array}{l}\text { Coal seam } \\
\text { thickness }(m)\end{array}$ & $\begin{array}{l}\text { Dip } \\
\text { angle }\left({ }^{\circ}\right)\end{array}$ & & & & \\
\hline \multirow[t]{3}{*}{1} & 3.21 & 1 & 0.9995 & 0.0376 & 0.0000 & $1,0,0$ \\
\hline & 2.78 & -1 & 0.0000 & 0.9058 & 0.0000 & $0,1,0$ \\
\hline & 3.48 & -7 & 0.0000 & 0.0000 & 1.0000 & $0,0,1$ \\
\hline \multirow[t]{3}{*}{2} & 3.25 & 1 & 0.9995 & 0.0376 & 0.0000 & $1,0,0$ \\
\hline & 3.20 & -3 & 0.0000 & 0.9946 & 0.0073 & $0,1,0$ \\
\hline & 3.15 & 7 & 0.0000 & 0.0024 & 0.9977 & $0,0,1$ \\
\hline \multirow[t]{3}{*}{3} & 3.22 & -7 & 0.9995 & 0.0376 & 0.0000 & $1,0,0$ \\
\hline & 3.40 & 1 & 0.0000 & 0.9783 & 0.4156 & $0,1,0$ \\
\hline & 3.25 & 7 & 0.0000 & 0.0000 & 1.0000 & $0,0,1$ \\
\hline
\end{tabular}

\section{Conclusions}

Small geological structures are well developed in the Zhangcun Coal Mine. Because these structures can weaken the rock strength and act as pathways for groundwater flow, they pose a threat to safe coal production. Although factors indicative of the presence of small geologic structures may vary, seven factors are to be considered and they are coal seam dip, thickness, the amount of gas gathering, inflow changes, temperature, fracture type and fragmentation degree of the coal seam. These factors are integrated into the backpropagation ANN model to predict the risk of three zones (normal zone, affected zone and failure zone). The ANN model can automatically simulate nonlinear, qualitative and quantitative relationships between the various factors, reduce man-made interference, and improve the effectiveness of the small structure prediction. On the basis of analyzing the controlling factors in the Zhangcun Coal Mine, two factors, the CSDA and CSTK, are screened out as the main factors controlling small structures. Twenty-six sets of data were collected with 23 as training data sets and the remaining 3 to verify the model performance. The prediction results of the model are in good agreement with the field test results. The distribution characteristics of the small structures can be accurately predicted in the coal seam extraction process as long as data of the controlling factors are accurate. Accurate prediction of the small faults and folds helps control hazards caused by gas and water in coal mines.

Acknowledgments This research was supported by the China National Science Foundation (Grant No. 41430318) and Research Fund for the Doctoral Program of Higher Education of China (Grant No. 20130023120018). The authors would like to thank the editor and the reviewers for their constructive suggestions.

\section{References}

Cai Y, Yang B, Sun H (1994) The application of artificial neural network to the prognosis of polymetallic deposits. Miner Depos 13(2):181-185 (in Chinese)
Cao L, Jiang Z (2002) Research on application of artificial neural network in predicting mining subsidence. J China Univ Min Technol 31(1):36-38 (in Chinese)

Chen P, Wu Q (2001) Fatalness assessment system of earth fissure based on artificial neural network. Coal Geol Explor 29(3):44-47 (in Chinese)

Li Z, Ma X (1993) Practical geological research in mine-method and progress. Geological Publishing House, Beijing (in Chinese)

Lv X, Zhao P (1998) Model of artificial neural networks for quantitative prediction of minerals. Earth Sci J China Univ Geosci 23(6):620-623 (in Chinese)

McCorak MD (1991) Neural computing in geophysics. Lead Edge 10(1):11-15

Wang W (1995) Introduction and application of artificial neural network. Beihang University Press, Beijing (in Chinese)

Wang G, Long R, Xu F (1993) Prediction of geological structure in coal mines. China Coal Industry Publishing House, Beijing (in Chinese)

Wu Q, Ye S (2008) The prediction of size-limited structures in a coalmine using artificial neural networks. Int J Rock Mech Min Sci 45(6):999-1006

Wu Q, Huang X, Dong D (1999) Application analysis of geographic information system on prediction of size-limited structures in the front of coal. J China Coal Soc 24(2):113-117 (in Chinese)

Wu Q, Yu J, Pang W (2007) Prediction of size-limited structures in the front of coal tunneling based on ANN. J China Univ Min Technol 36(4):446-452 (in Chinese)

Wu Q, Xu H, Pang W (2008) GIS and ANN coupling model: an innovative approach to evaluate vulnerability of karst water inrush in coalmines of north China. Environ Geol 54(5):937-943

Wu Q, Liu Y, Yang L (2011) Using the vulnerable index method to assess the likelihood of a water inrush through the floor of a multi-seam coal mine in China. Mine Water Environ 30(1):54-61

Wu Q, Liu Y, Luo L (2015) Quantitative evaluation and prediction of water inrush vulnerability from aquifers overlying coal seams in Donghuantuo Coal Mine, China. Environ Earth Sci 74:1429-1437

Yang Z (1997) The problem of using fault strike to predict the small and medium structure in coal seam. J Hebei Coal 3:54-55 (in Chinese)

Zeng Y, Wu Q (2014) Minor structure prediction ahead of coal roadway advance based on ANN technology in Jining No. 2 Coalmine. Coal Geol China 26(9):13-16 (in Chinese) 